\title{
Exome sequencing identifies a novel TTC37 mutation in the first reported case of Trichohepatoenteric syndrome (THE-S) in South Africa
}

Craig Kinnear ${ }^{{ }^{*}}$ D, Brigitte Glanzmann ${ }^{1}$, Eric Banda ${ }^{1}$, Nikola Schlechter ${ }^{1}$, Glenda Durrheim ${ }^{1}$, Annika Neethling ${ }^{1}$, Etienne $\mathrm{Nel}^{3}$, Mardelle Schoeman', Glynis Johnson', Paul D. van Helden', Eileen G. Hoal', Monika Esser², Michael Urban ${ }^{1}$ and Marlo Möller ${ }^{1}$

\begin{abstract}
Background: Trichohepatoenteric syndrome (THE-S) or phenotypic diarrhoea of infancy is a rare autosomal recessive disorder characterised by severe infantile diarrhoea, facial dysmorphism, immunodeficiency and woolly hair. It was first described in 1982 in two infants with intractable diarrhoea, liver cirrhosis and abnormal hair structure on microscopy. We report on two siblings from a consanguineous family of Somali descent who, despite extensive clinical investigation, remained undiagnosed until their demise. The index patient died of fulminant cytomegalovirus pneumonitis at 3 months of age.

Methods: Whole exome sequencing (WES) was performed on a premortem DNA sample from the index case. Variants in a homozygous recessive state or compound heterozygous state were prioritized as potential candidate variants using TAPER ${ }^{\mathrm{TM}}$. Sanger sequencing was done to genotype the parents, unaffected sibling and a deceased sibling for the variant of interest.

Results: Exome sequencing identified a novel homozygous mutation (c.4507C > T, rs200067423) in TTC37 which was confirmed by Sanger sequencing in the index case. The identification of this mutation led to the diagnosis of THE-S in the proband and the same homozygous variant was confirmed in a male sibling who died 4 years earlier with severe chronic diarrhoea of infancy. The unaffected parents and sister were heterozygous for the identified variant.

Conclusions: WES permitted definitive genetic diagnosis despite an atypical presentation in the index case and suggests that severe infection, likely secondary to immunodeficiency, may be a presenting feature. In addition definitive molecular diagnosis allows for genetic counseling and future prenatal diagnosis, and demonstrates the value of WES for post-mortem diagnosis of disorders with a non-specific clinical presentation in which a Mendelian cause is suspected.
\end{abstract}

Keywords: Trichohepatoenteric syndrome, Primary immunodeficiency diseases, Exome, Consanguineous, Next generation sequencing, Sanger sequencing

\footnotetext{
* Correspondence: gkin@sun.ac.za

${ }^{1}$ SA MRC Centre for Tuberculosis Research, DST/NRF Centre of Excellence for Biomedical Tuberculosis Research, Division of Molecular Biology and Human Genetics, Faculty of Medicine and Health Sciences, Stellenbosch University, P.O. Box 241, Cape Town 8000, South Africa

Full list of author information is available at the end of the article
} 


\section{Background}

Trichohepatoenteric syndrome (THE-S) is a rare autosomal recessive disorder characterised by severe infantile diarrhoea, intrauterine growth retardation (IUGR), hair abnormalities (mainly trichorrhexis nodosa), skin abnormalities (including café-au-lait macules), hepatopathy and immunodeficiency [1-5]. All affected children, who present with explosive diarrhoea, require parenteral nutrition for survival. In some instances parenteral nutrition is a life-long requirement, while in others the patient can be weaned to full enteral feeding [6]. Fine motor movement abnormalities and mental retardation have also been reported in some affected individuals [7]. The estimated prevalence of THE-S is approximately $1 /$ 400,000-500,000 live births [6] and since its first description in 1982 [1], less than 60 cases have been described, with cases being reported from Europe, Asia, the Middle East and North Africa.

The diagnosis should be suspected in the presence of the clinical triad of IUGR, severe protracted diarrhoea of early onset, and abnormal facial features with prominent ears and cheeks [7]. Additional features are often present but are less consistent. Even though these key syndromic features have been very well described, the age-related appearance of many of these features coupled with the estimated low prevalence of this disease make it challenging to recognize THE-S during early infancy [3, 7]. It should be noted that before the hair abnormalities, liver cirrhosis or facial dysmorphism become noticeable, many patients fail to thrive, and suffer from intractable diarrhoea and recurrent infections, which are three of the 10 warning signs of primary immunodeficiency diseases (PIDs) [8-11]. For these reasons, PIDs are often initially suspected in THE-S patients. In a review of the literature, Fabre and colleagues noted that 39 of 44 reported cases presented with an immunological defect [12]. These immunological deficits ranged from low serum immunoglobulin levels [12], deficits in antibody production following vaccinations [6], monoclonal hyper IgA [13] and low lymphocyte counts $[1,3]$.

While our understanding of the pathogenesis of THE$S$ remains limited, causal mutations in two genes, TTC37 [6] and SKIV2L [7, 12, 14-16], both encoding components of the human Ski complex, have been reported. The Ski complex is an obligatory co-factor in the RNA exosome which is crucial for the accurate processing of nuclear RNA precursors and in the degradation of RNA in both the nucleus and the cytoplasm [17-19] . Most reported mutations are private and are distributed in all exons of TTC37 and SKIV2L, but a founder mutation in TTC37 (c.2808G >A) has been reported in patients from India [5].

Interestingly, one case of THE-S presenting with immunodeficiency without diarrhoea has recently been reported by Rider and co-workers [15]. Their study described a novel TTC37 mutation in a patient who presented with frequent otitis media, viral infections, purulent conjunctivitis, woolly hair and distinctive facial features without the characteristic severe diarrhoea of infancy seen in THE-S [15].

Here we describe a patient with an atypical respiratory presentation in which exome sequencing identified a novel homozygous TTC37 mutation and allowed the diagnosis of THE-S to be made.

\section{Methods}

\section{Case description}

The index patient was a male of Somalian descent, born to consanguineous parents (first cousins once-removed, i.e. fourth degree relatives) living in South Africa (see Fig. 1 for pedigree). He was born at 35 weeks gestation, weighing $1940 \mathrm{~g}$ (3rd percentile) and remained in hospital initially for three weeks for neonatal jaundice and poor weight gain. The parents noted subtle patchy hyperpigmentation after he completed phototherapy for jaundice. His hair was shaved per their custom and was reportedly not unusual. On discharge he had received all routine vaccinations including live BCG and oral Polio drops.

At age three months the patient presented with an acute history of respiratory distress. In addition, he had failed to thrive and his mass was $2600 \mathrm{~g}(<<3$ rd centile for corrected age). There was clinical evidence of a pneumonia and the liver edge was palpable $2 \mathrm{~cm}$ below the costal margin. Patchy, poorly circumscribed areas of hyperpigmentation were present on the trunk and limbs (Fig. 2a). There was an exfoliating rash particularly on the hands and feet with erythema of the palms and soles suggestive of erythroderma (Fig. 2b). The forehead was prominent (Fig. 2c), giving a triangular appearance to the face, but no hypertelorism or other dysmorphic features were noted.

An echocardiogram was performed and found to be normal, while chest $\mathrm{x}$-ray showed an extensive bilateral interstitial pneumonitis with alveolar changes (Fig. 2d). The C-reactive protein (CRP) increased over 3 days from 9 to $43 \mathrm{mg} / \mathrm{L}$ (ref: $0-10$ ). Tracheal aspirate was positive for cytomegalovirus (CMV) but not other respiratory viruses, and CMV viraemia was documented with a viral load of $\log 5.97$. Blood, sputum and stool culture showed no evidence of bacterial infection. The full blood count was normal, with normal number of lymphocytes. On subset analysis, the natural killer (NK) cell count was 81/microliter (ref: 300-700) and the NK cell percentage was 3\% (ref: 8-17) (Table 1). There was also a low serum IgG levels of $<1.41 \mathrm{~g} / \mathrm{L}$ (ref 3.00-10.00) but normal IgA and IgM. Liver functions showed very elevated gammaglutamyl transferase (942 IU/L, ref: 2-30); somewhat elevated conjugated bilirubin (10 micromol/L, ref: 0-6), 


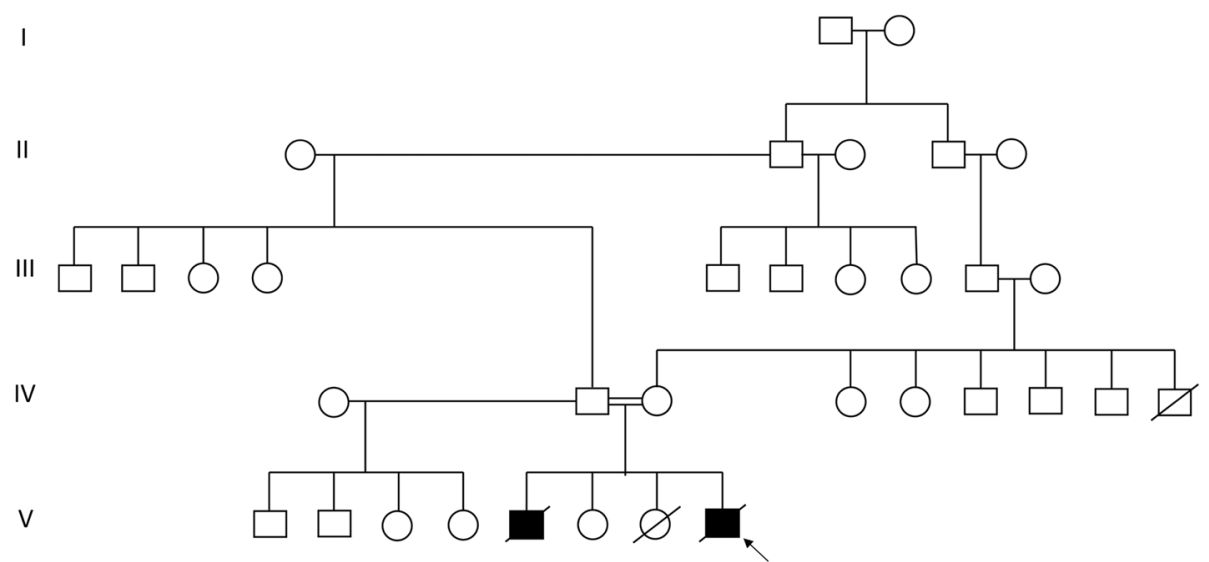

Fig. 1 Multigeneration pedigree of the Trichohepatoenteric syndrome family

alanine transaminase (39 IU/L, ref: 4-35) and aspartate transaminase (75 IU/L, ref: 0-65); low albumin (23 g/L, ref: $28-46)$, and normal alkaline phosphatase (180 IU/L, ref: 75-316). The plasma amino acid profile was normal, as were urinary organic acids. Skin biopsy histology of a hyperpigmented macule showed abundant melanin but no specific features for pathological processes such as graft versus host disease.

The patient was treated in the intensive care unit. Treatment included ventilation with a high frequency oscillator, intravenous ganciclovir, and other medications as appropriate. During the admission diarrhoea was noted, but was not a prominent feature. Following three weeks of intensive therapy, the patient died of ongoing severe pneumonitis. Supervening nosocomial sepsis was suspected but unproven. The family declined an autopsy. The clinical suspicion was of a combined immune deficit, with a family history that suggested an autosomal recessive or perhaps X-linked disorder.
A male sibling born 4 years earlier, weighed $1840 \mathrm{~g}$ $(<<3$ rd centile $)$ at term gestation. He was treated for neonatal jaundice and subsequently readmitted at two months of age with chronic diarrhoea, dehydration and failure to thrive. He was noticed to have hair that was "sticking out", and both hyper- and hypopigmented skin macules. Other clinical features included a prominent forehead with a triangular face and low-set ears, penile hypospadias and bilateral camptodactyly of the fingers. Further laboratory investigations confirmed a secretory diarrhoea with albumin loss and metabolic acidosis. Endoscopic biopsies of the stomach and duodenum were performed. The stomach histology was normal, whereas the duodenal biopsy showed flattening and thickening of some villi, and preserved crypt architecture; in addition the lamina propria contained a sprinkling of lymphocytes and plasma cells. The liver function test was only minimally deranged. The full blood count, lymphocyte subsets and serum immunoglobulin profile were similar
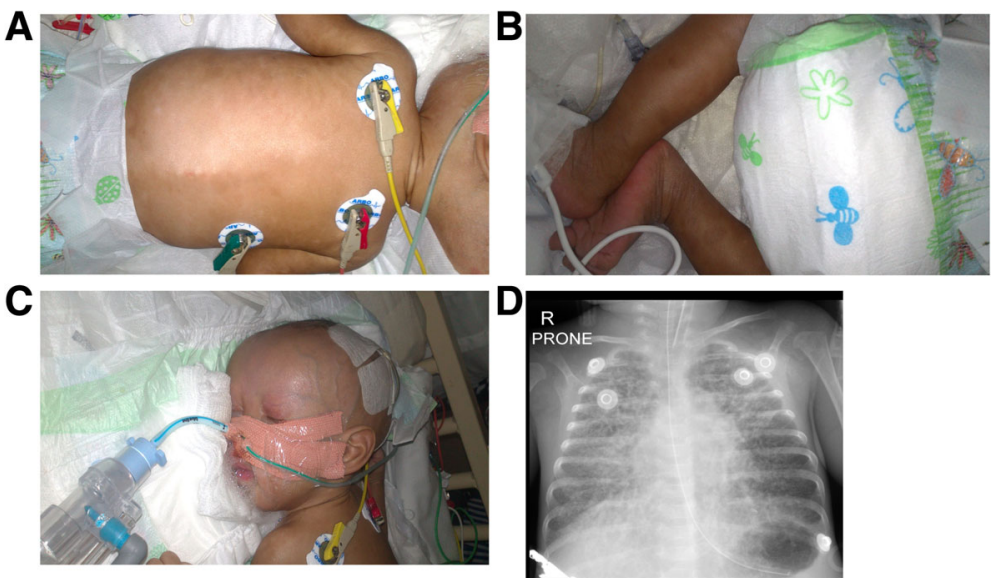

Fig. 2 Clinical features of the proband. a Hyperpigmentation of the trunk. $\mathbf{b}$ Exfoliating rash present at the feet of the proband. $\mathbf{c}$ Prominent forehead with triangular appearance of the face. $\mathbf{d}$ Chest $\mathrm{X}$-ray showing extensive bilateral bronchopneumonia 
Table 1 Phenotypic comparison of both siblings to literature

\begin{tabular}{|c|c|c|c|}
\hline Clinical feature (HPO id) & Index case & Affected brother & $\begin{array}{l}\text { Findings in } 25 \text { previously reported } \\
\text { cases }[1,3,4,6,15]\end{array}$ \\
\hline Consanguinity & Y & Y & 13/22 (59\%) \\
\hline IUGR (HP:0001511) & Y & Y & $23 / 25(92 \%)$ \\
\hline Preterm <37 wk (HP:0001622) & Y & N & $12 / 25(48 \%)$ \\
\hline \multicolumn{4}{|l|}{ Dysmorphism } \\
\hline Prominent forehead (HP:0011220) & Y & Y & 20/20 (100\%) \\
\hline Hypertelorism (HP:0000316) & N & N & $20 / 20(100 \%)$ \\
\hline Hypo/pigmented skin rash +/- erythroderma & Y & Y & 6/20 (30\%) \\
\hline $\begin{array}{l}\text { Unusual hair (Includes trichorrhexis nodosa, } \\
\text { HP:0009886) }\end{array}$ & Unknown (shaved) & Y ('sticking out') & $20 / 20(100 \%)$ \\
\hline $\begin{array}{l}\text { Congenital heart defect (Abnormal heart } \\
\text { morphology, HP:0001627) }\end{array}$ & N & N & $5 / 20(25 \%)$ \\
\hline $\begin{array}{l}\text { Musculoskelelal abnormality (Camptodactyly } \\
\text { HP:0005617) }\end{array}$ & N & Y (camptodactyly) & 1/12 (8\%; Perthes disease) \\
\hline Urinary tract abnormality (Hypospadias, HP:0000047) & N & Y (hypospadias) & $3 / 20(15 \%)$ \\
\hline \multicolumn{4}{|l|}{ Gastrointestinal } \\
\hline Chronic diarrhea (HP:0002028) & N & Y & $24 / 25(96 \%)$ \\
\hline Failure to thrive (HP:0001508) & Y & Y (needed TPN) & $25 / 25(100 \%)$ \\
\hline $\begin{array}{l}\text { Villous atrophy on biopsy of small } \\
\text { intestine (HP:0011473) }\end{array}$ & - & Y (patchy) & $21 / 21(100 \%)$ \\
\hline Liver dysfunction or fibrosis & N & N & 9/17 (53\%, all with liver fibrosis) \\
\hline \multicolumn{4}{|l|}{ Outcome } \\
\hline Neurodevelopmental delay (HP:0012758) & $?$ & Y & 10/16 (63\%) \\
\hline Death & Y & Y & $12 / 25(48 \%)$ \\
\hline If deceased, age at death & 3 months & 5 months & 2-96 months \\
\hline \multicolumn{4}{|l|}{ Immunological investigations \{reference range\} } \\
\hline Lymphocyte count & $\left.2.53\left\{2.0-17.0 \times 10^{9} /\right\}\right\}$ & $4.17\left\{2.0-17.0 \times 10^{9} / 1\right\}$ & \multirow[t]{2}{*}{ Where reported was normal } \\
\hline \% B-cells (CD19) & $24 \%\{19-31 \%\}$ & $20 \%\{19-31 \%\}$ & \\
\hline \% T-cells (CD3) & $73 \%\{58-67 \%\}$ & $72 \%\{58-67 \%\}$ & \multirow[t]{4}{*}{$\begin{array}{l}\text { Weak T-cell proliferative response in } \\
\text { some cases to antigens, not mitogens }\end{array}$} \\
\hline \% T-helper cells (CD4) & $55 \%\{38-50 \%\}$ & $39 \%\{38-50 \%\}$ & \\
\hline \% T-supressor cells (CD8) & $16 \%\{18-25 \%\}$ & $33 \%\{18-25 \%\}$ & \\
\hline $\begin{array}{l}\text { \% Natural killer cells }(C D 16,56) \text { (Abnormality } \\
\text { of NK cells HP:0012176) }\end{array}$ & $3 \%\{8-17 \%\}$ & $8 \%\{8-17 \%\}$ & \\
\hline IgG level (IgG deficiency HP: 0004315) & $<1.41 \mathrm{~g} / \mathrm{l}\{3.0-10.0 \mathrm{~g} / \mathrm{l}\}$ & $1.96 \mathrm{~g} / \mathrm{l}\{3.0-10.0 \mathrm{~g} / \mathrm{l}\}$ & \multirow{3}{*}{$\begin{array}{l}11 / 20 \text { (55\%) had 'low immunoglobulins'. } \\
\text { Other often had deficient vaccine response. } \\
\text { Hence THE-S now in antibody deficiency } \\
\text { group of the } 2015 \text { IUIS Primary } \\
\text { Immunodeficiency classification }\end{array}$} \\
\hline IgA level (IgA deficiency HP:0002720) & $0.44 \mathrm{~g} / \mathrm{l}\{0.1-0.7 \mathrm{~g} / \mathrm{l}\}$ & $<0.25 \mathrm{~g} / 1\{0.1-0.7 \mathrm{~g} / \mathrm{l}\}$ & \\
\hline IgM level & $0.92 \mathrm{~g} / \mathrm{l}\{0.2-1.10 \mathrm{~g} / \mathrm{l}\}$ & $1.19 \mathrm{~g} / \mathrm{l}\{0.2-1.10 \mathrm{~g} / \mathrm{l}\}$ & \\
\hline
\end{tabular}

HPO id human phenotype ontology identification number, $Y$ Yes, N No, TPN total parenteral nutrition, NK natural killer, IUGR intrauterine growth restriction, IUIS International Union of Immunological Societies

to that of the index case, except that the IgA was also low. He remained in hospital until his demise at 5 months of age, following documented episodes of Klebsiella and Enterococcal septicaemia. Additional investigations showed a normal karyotype, and normal profile of urine organic acids and urine and blood amino acids. No definitive diagnosis was made, and some details only became available to us after the death of the index case. Phenotypic comparisons between the index case and his deceased brother are shown in Table 1. The couple also has a healthy daughter, and a female stillbirth for reasons which were considered to be unrelated (Fig. 1).

Prior to demise of the index case, the parents were counselled on a possible genetic immunodeficiency and consented for blood samples from all live members of the family to be submitted for exome sequencing. 
The study was approved by the Health Research Ethics Committee of Stellenbosch University (study no. N13/ 05/075). Parents gave informed consent to participate in the study, which included the genetic evaluation of their children. Additional informed consent was obtained from the parents to include photographs in this article. The study adhered to the ethical guidelines as set out in the "Declaration of Helsinki, 2013" [20]. Venous blood for DNA extraction and WES was drawn from the index case $(1 \mathrm{ml})$ and both of his parents $(5 \mathrm{ml})$. Blood was also drawn from the unaffected sister for genetic analysis. DNA was purified from blood using the Nucleon BACC3 Kit (Amersham Biosciences, Buckinghamshire, UK). DNA from a formalin-fixed duodenal biopsy sample of the previously deceased male sibling was extracted using the QIAamp ${ }^{\circledR}$ DNA FFPE Tissue Kit (Qiagen, Hilden, Germany).

\section{Exome capture and sequencing}

Library preparation for sequencing was carried out using the Ion AmpliSeq ${ }^{\text {TM }}$ Exome RDY Kit and the Ion Xpress ${ }^{\text {TM }}$ Barcode Adaptors 1-16 Kit (Life Technologies, Carlsbad, California, United States). The DNA template for sequencing was prepared on the Ion Chef system using the Ion PI ${ }^{\mathrm{TM}} \mathrm{Hi}-\mathrm{Q}^{\mathrm{TM}}$ Chef Kit and the Ion PI ${ }^{\mathrm{mM}}$ Chip Kit v3. Sequencing was carried out on the Ion Proton ${ }^{\text {TM }}$ (Thermo Fisher, Carlsbad, California, United States) at the Centre for Proteomic and Genomic Research (CPGR), Observatory, Cape Town, South Africa.

\section{Read mapping, variant detection and functional annotation}

Sequences were aligned to the human reference genome, hg19 using Torrent Mapping Alignment Program (TMAP, version 4.4.11-1) in the ion-analysis workflow on the Torrent Suite (version 4.4.3). Base quality score recalibration, indel realignment and variant calling were performed using the variantCaller (version 4.4.3.3) plugin on the Torrent Suite and variant annotation was performed. Variant prioritization was performed using TAPER ${ }^{\mathrm{TM}}$, a custom-designed, in-house method and variants in a homozygous recessive state or compound heterozygous state were prioritized as potential candidate variants [21]. In brief, TAPER $^{\mathrm{TM}}$ 1) submits VCF files to an online variant caller, 2) removes all synonymous and non-frameshift indel variants, 3) removes all variants with a frequency of greater than $1 \%$ if present in the 1000 Genomes Project or Exome Sequencing Project 6500,4) removes all variants with negative Genomic Evolutionary Rate Profiling (GERP) +++ scores, 5) removes all variants with Functional Analysis through Hidden Markov Models (FATHMM) scores greater than $0.1,6$ ) removes $\mathrm{X}$ and $\mathrm{Y}$ chromosome variants (if the pedigree does not indicate sex-linked inheritance) and lastly 7) identifies associated disorders for prioritised genes. Additionally, to validate findings obtained using $\mathrm{TAPER}^{\mathrm{T \mu}}$, variant filtering and prioritization was also done using Ion Reporter ${ }^{\mathrm{TM}}$ Software (version 5.0).

Analysis of identity-by-descent and runs of homozygosity Using custom scripts, the VCF files for both the mother and father were merged to create a multi-sample VCF file. This file was subsequently filtered for single nucleotide substitutions that were flagged "PASS" by a custom variant caller filter. Identity by descent (IBD) was calculated uing PLINK (http://zzz.bwh.harvard.edu/plink/) [22]. IBD was estimated in order to determine the degree of relatedness across the two individuals (PI(HAT)). This is calculated by using the inferred probabilities of individuals sharing an allele. Individuals may therefore share no alleles, one allele or both alleles. Following IBD, the runs of homozygosity were calculated to identify which segments of specific chromosomes are identical to one another. This allowed further inference of IBD as these segments were identical as a result of inheritance as opposed to sequence similarity.

\section{Sanger sequencing}

A 239 base pair fragment containing the candidate variant was polymerase chain reaction (PCR)-amplified from genomic DNA of the index case, his affected brother, his unaffected parents and his unaffected sister using the following primers: TTC37F- 5' AA TCATAATCAGACACTACATCTGC-3' and TTC37R- 5' GCTCATAGTCATCTTTGGCATATAA. Each amplicon was bi-directionally sequenced using the BigDye ${ }^{\oplus}$ Terminator v3.1 Cycle Sequencing Kit (Perkin-Elmer, Applied Biosystems Inc., Foster City, California, USA.), followed by electrophoresis on an ABI 3130XL Genetic Analyzer (Perkin-Elmer, Applied Biosystems Inc., Foster City, California, USA). All automated DNA sequencing reactions were performed at the Central Analytical Facility at Stellenbosch University, Stellenbosch, RSA.

\section{Results}

IBD and segmental sharing were calculated between the two parents of the index case. A PI(HAT) score of 0.082765 (Table 2) was calculated for the parents of the proband. This is within the range of what would be expected for first degree cousins. Moreover, eight runs of

Table 2 Identity by descent scores (IBD) between the mother and father of the index case

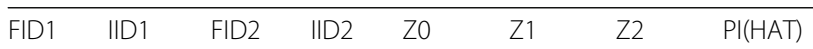

PID027 Mother PID027 Father $0.053790 .04621 \quad 0.07311 \quad 0.082765$

FID Family ID, IID Individual ID, ZO probability that individuals at a specific marker will share no alleles, $Z 1$ probability that individuals at a specific marker will share 1 allele, $Z 2$ probability that individuals at a specific marker will share 2 alleles 
homozygosity across six chromosomes were identified in the proband (Table 3 ).

The summary of the exome sequencing data for the index case and both his parents are presented in Table 4 . Following filtering for rare or unreported variants a total of seven rare, homozygous variants were identified (Table 5). Following further analysis and database mining for gene-disease association, we identified a rare, low frequency homozygous missense mutation c. 4507C $>T$ (rs200067423) in exon 42 of TTC37 (NM_014639) which results in the substitution of an arginine with a cysteine at amino acid 1503 (p.R1503C) as a possible disease-causing variant. This is the first time that this variant has been identified in homozygous form and the read depth of this variant was $43 \mathrm{X}$. This variant was found in one of the eight identified runs of homozygosity (Table 3). Additionally, using the American College of Medical Genetics this variant is classified as likely pathogenic pathogenic (One moderate and four supporting; PM2, PP1, PP2, PP3 and PP4) [23]. No other known disease-causing mutations were identified in TTC37 or SKIV2L, both of which were $100 \%$ covered by WES. Additionally, no disease-causing mutation was identified on the $\mathrm{X}$ chromosome (Additional file 1: Tables S1 and S2).

The presence of the variant in the patient and family members was verified using Sanger sequencing. The details of the mutation are summarised in Table 6. In silico predictions of the pathogenicity of the TTC37 c. $4507 C>T$ mutation (SIFT, Polyphen) found that this mutation is damaging (Table 5). Multiple sequence alignment between species showed that the mutation occurs at a highly conserved residue (Fig. 3). Both parents as well as the healthy sister were heterozygous for the mutation, while the previously deceased sibling was homozygous (Fig. 4). This is the first reported case of THE-S in a patient of Somali descent as well as the first reported case in South Africa.
Table 4 Summary of exome sequencing data for the index case and his parents

\begin{tabular}{llll}
\hline & Proband & Mother & Father \\
\hline Total captured regions size & $64 \mathrm{Mb}$ & $64 \mathrm{Mb}$ & $64 \mathrm{Mb}$ \\
\% of captured regions with coverage $>10$ & 99.7 & 99.7 & 99.8 \\
Average coverage of captured region (\%) & 98.8 & 98.6 & 99.0 \\
Total number of SNPs & 21,858 & 22,433 & 22,043 \\
Total number of INDELs & 510 & 503 & 515 \\
N rare homozygous & 23 & 11 & 16 \\
N rare heterozygous & 269 & 201 & 201 \\
N X linked & 21 & 15 & 18 \\
\hline
\end{tabular}

SNP single nucleotide polymorphism

\section{Discussion}

THE-S is an extremely rare autosomal recessive disorder that typically manifests with severe, explosive diarrhoea in infancy, hair abnormalities, dysmorphic facial features and immunodeficiency. Here we describe brothers with a rare homozygous TTC37 mutation (not previously described in the homozygous state), and presenting with many THE-S clinical features, but an atypical presentation in the index case. Due to this atypical presentation, and the lack of some of the specific features, THE-S was not initially considered. The characteristic hair abnormalities were not observed because his head was shaven. In addition, THE-S has not been previously diagnosed in South Africa, where consanguinity is uncommon.

The sibling of the index case had a more typical gastrointestinal presentation for THE-S, but the details of the case were not immediately available. In addition, the presence of hypospadias and camptodactyly would suggest consideration of other diagnostic possibilities. As a result, although a Mendelian disorder, either autosomal recessive or X-linked, was strongly suspected, the differential diagnosis remained broad despite quite extensive laboratory investigation.

Table 3 The identification of runs of homozygosity between the mother and father of the index case

\begin{tabular}{llllllllll}
\hline CHR & SNP1 & SNP2 & POS1 & POS2 & KB & NSNP & DENSITY (KB/NSNP) & PHOM & PHET \\
\hline 1 & rs6077208 & rs773530539 & 21798208 & 23743698 & 1945.49 & 116 & 16.771 & 0.603 & 0.017 \\
2 & rs558321757 & rs23253874 & 25050977 & 33764286 & 8713.30 & 236 & 36.920 & 0.674 & 0.038 \\
2 & rs773745425 & rs83697568 & 178415694 & 180846743 & 2431.04 & 123 & 19.764 & 0.789 & 0.008 \\
$\mathbf{5}$ & rs107007917 & rs70038334 & $\mathbf{9 0 7 1 9 1 2 9}$ & $\mathbf{9 4 9 5 3 3 7 9}$ & $\mathbf{4 2 3 4 . 2 5}$ & $\mathbf{1 5 1}$ & $\mathbf{2 8 . 0 4 1}$ & $\mathbf{0 . 3 1 4}$ & $\mathbf{0 . 5 0 8}$ \\
6 & rs7451498 & rs9384046 & 148981394 & 153173999 & 4192.60 & 163 & 25.721 & 0.662 & 0.131 \\
14 & rs10134181 & rs898927 & 92280037 & 98263147 & 5983.11 & 244 & 24.520 & 0.297 & 0.637 \\
16 & rs4785195 & rs1004299 & 49932806 & 54343840 & 4411.03 & 129 & 34.194 & 0.676 & 0.180 \\
16 & rs990813 & rs12596363 & 59070616 & 64124789 & 5054.17 & 117 & 43.198 & 0.088 & 0.124 \\
\hline
\end{tabular}

Bold font denotes $\mathrm{ROH}$ in which TTC37 is localized. All calculations were done using the VCF file obtained for the WES. Stringent quality control parameters were implemented before identity by decent (IBD) and ROH were calculated. Note that because of missing calls, PHOM + PHET $<1$

SNP1 start of the SNP segment, SNP2 end of the SNP segment, POS1 start of the physical position of the segment (base pair), POS2 end of the physical position of the segment (base pair), NSNP number of SNPs in the segment, KB physical length of the segment, PHOM proportion of sites homozygous, PHET proportion of sites heterozygous 


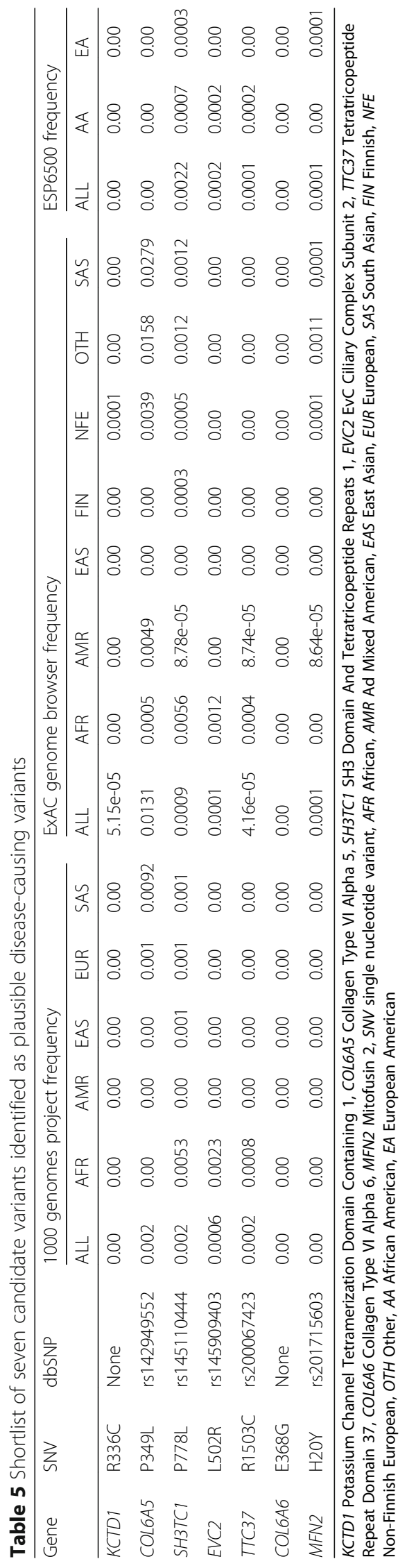


Table 6 Details of the candidate variant narrowed down using consecutive filters based on an autosomal recessive model of inheritance and low frequency

\begin{tabular}{|c|c|}
\hline Chromosome & Chr 5 \\
\hline Position & 94803683 \\
\hline Gene name & TTC37 \\
\hline RefSeq & NM_014639 \\
\hline Reference sequence & G \\
\hline Proband: number of reads with reference & 0 \\
\hline Proband: alternative & A \\
\hline Proband: number of reads with alternative & 43 \\
\hline Mother: number of reads with reference & 88 \\
\hline Mother: alternative & A \\
\hline Mother: number of reads with alternative & 43 \\
\hline Father: number of reads with reference & 49 \\
\hline Father: alternative & A \\
\hline Father: number of reads with alternative & 11 \\
\hline Mutation type & Missense \\
\hline Mutation: DNA (HGVS nomenclature_c.) & c.4597 C > T \\
\hline Mutation: protein (HGVS nomenclature_p.) & R1503C \\
\hline Prediction $<$ SIFT & Damaging \\
\hline Prediction < PolyPhen-2 & Probably damaging \\
\hline Sanger verification & Yes \\
\hline
\end{tabular}

HGVS Human Genome Variation Society, SIFT Sorting Intolerant from Tolerant

Whole exome sequencing, conducted to clarify the clinical diagnosis, revealed a rare homozygous missense c. 4507C > T mutation (rs200067423) in TTC37, one of two genes previously implicated in THE-S in several independent investigations. The patient was found to be homozygous for the mutation while each of his consanguineous parents and his unaffected sister were heterozygous. We also identified the same homozygous mutation in his affected brother. This mutation results in a substitution of an arginine, which is a hydrophilic positively charged amino acid, with a hydrophobic uncharged cysteine residue at amino acid position 1503 of the protein. Based on the differences in charge and hydrophobicity between the arginine and cysteine and the fact that cysteine has the potential to form disulphide bonds, it is highly likely that the mutation leads to the loss of hydrogen bonds or impairs the proper folding of TTC37 [24]. Additionally, multiple species alignment shows that the mutation occurs at a highly conserved residue.

The findings on history, examination and laboratory investigations in both affected boys are consistent with a diagnosis of THE-S, despite the atypical presentation in the index case. IUGR is described as a consistent finding [7] and was present in both affected boys. The pre-deceased brother had chronic secretory diarrhoea with onset in early infancy, with associated failure to thrive, as described in the original article by Stankler et al.[1]. In the index case diarrhoea was present but less prominent than the severe respiratory infection.

On examination, a wide and prominent forehead is a consistent finding in THE-S [6] and was present in both cases. Skin changes occur frequently and include erythroderma [3] and patchy skin hyperpigmentation [7]. The index case had erythroderma and both had patchy hyperpigmentation. Trichorrhexis nodosa was not formally assessed, but the earlier sibling was known to have unusual-looking scalp hair. Both had neonatal jaundice and the index case had evidence of ongoing liver dysfunction, which is a common finding in THE-S [6]. The findings on duodenal histology for the earlier sibling were not very specific, but were consistent with previously reported findings in THE-S. Taken together, the results of the exome sequencing in the index case and the clinical findings in both affected siblings confirm a diagnosis of THE-S.

The fulminant CMV pneumonitis in the index case, taken together with the abnormal immunological profile, suggests underlying primary immunodeficiency. While humoral immune deficits are known to be common in THE-S $[6,14]$, the low natural killer (NK) cell count, more marked in the index case, has not been previously described. NK cells are important in recognizing and killing virally infected cells, and deficiency predisposes specifically to severe infections with herpes viruses such as CMV [25]. This provides a possible

$$
\begin{aligned}
& \text { Zebrafish GEVLKMSKSPVALLFOALLOFSVKMGARETR R LLERIVYASALGGSETIASVARWYLLRH } 1549 \\
& \begin{array}{llll}
\text { Mouse } & \text { SEALKVCFSPLAVELQALLQFNRKMGARETR } & \text { R } & \text { LLERIVYQTGY--PSSIVSAARWYLLRH } 1528
\end{array} \\
& \begin{array}{ll|l|l}
\text { Rat } & \text { SEALKVCFSPLAIFLQALLQFNRKMGARETR } & \text { R } & \text { LLERVVYQTGY--PNSIVSTARWYLLRH } 1528
\end{array} \\
& \text { Dolphin TEALKLCFCPLAVLLQALLQFKRKMGARETR } \mathrm{R} \text { LLERVVYQPGY--PKSIVSTARWYLLRH } 1522
\end{aligned}
$$

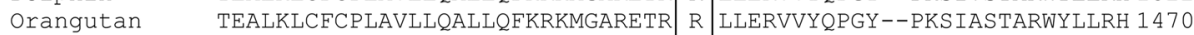

$$
\begin{aligned}
& \begin{array}{ll|l|l}
\text { Human } & \text { TEALKLCFCPLAVLLQALLQFKRKMGARETR } & \text { R } & \text { LLERVVYQPGY--PKSIASTARWYLLRH } 1529
\end{array}
\end{aligned}
$$

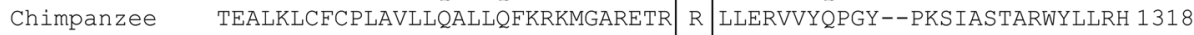

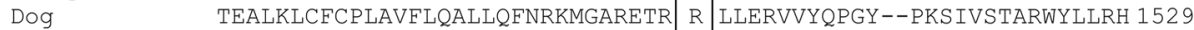

$$
\begin{aligned}
& \begin{array}{l|l|l}
\text { COW AEALKLCFCPLAVLLQALLQFKRKMGARETR } & \text { R } & \text { LLERVVYQPGY--PKSIVSTARWYLLRH } 1530
\end{array}
\end{aligned}
$$

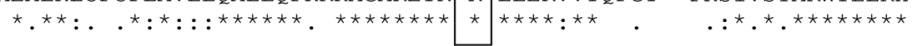

Fig. 3 ClustalW multiple sequence alignment showing the highly conserved position of the C.4507C > T (p.1053R > C) mutation in TTC37 


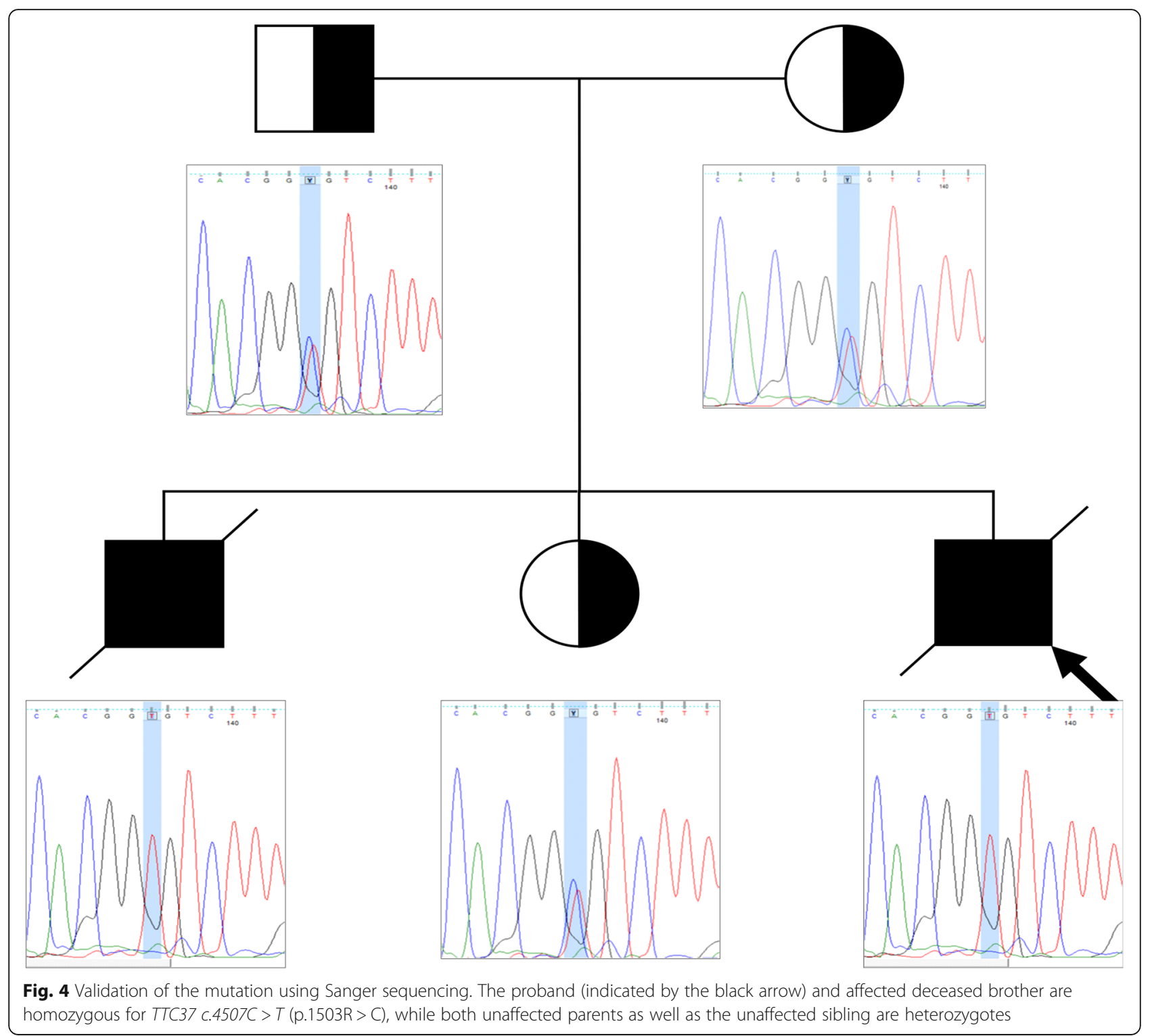

explanation for the severity of the CMV pneumonitis. Although childhood exposure to CMV is common, we found two prior cases of CMV hepatitis in previous reports of THE-S [3, 4], one of which occurred shortly after liver transplantation and therefore perhaps was secondary to use of immunosuppressants [4]. The predisposition to severe CMV may not be common in THE-S, but an alternative possibility is that THE-S is undetected in cases with early death.

\section{Conclusions}

WES has been available for several years, but its usefulness as a routine clinical diagnostic tool is only now beginning to emerge [26, 27]. WES has particular value in diagnosing the cause of rare conditions with unclear phenotypes [28] or with an atypical presentation [29] as found in our index case. We demonstrate how WES, together with detailed phenotyping of a previously and more typically affected family member, led to a postmortem molecular diagnosis of THE-S in an atypical index case. This allowed for genetic counselling and potential further genetic testing in the family; for carrier status, diagnosis or prenatal diagnosis. The findings in the index case broaden the range of phenotypes associated with THE-S to include presentation with fulminant CMV pneumonitis, and low NK cell count. We recommend that immunological investigations for known or suspected cases of THE-S include detailed immune function testing of antibody related function, but also careful screening for viral infections [10]. 


\section{Additional file}

Additional file 1: Supplementary Tables. (DOCX 13 kb)

\section{Abbreviations}

BCG: Bacillus Calmete-Guérin; CMV: Cytomegalovirus; CRP: C-reactive protein; DNA: Deoxyribonucleic acid; FATHMM: Functional Analysis through Hidden Markov Models; GERP: Genomic Evolutionary Rate Profiling; IBD: Identity by descent; IgA: Immunoglobulin A; IgG: Immunoglobulin G; IgM: Immunoglobulin M; IUGR: Intrauterine growth retardation; NK: Natural killer; PCR: Polymerase chain reaction; PID: Primary immunodeficiency diseases; SIFT: Sorting Intolerant From Tolerant; SKIV2L: Ski2 Like RNA Helicase; TAPER: Tool for Automated selection and Prioritization for Efficient Retrieval of sequence variants; THE-S: Trichohepatoentric syndrome; TTC37: Tetratricopeptide Repeat Domain 37; VCF: Variant call format; WES: Whole exome sequencing

\section{Acknowledgements}

Our gratitude goes to the family who consented to take part in this study.

\section{Funding}

This work was supported by the National Research Foundation of South Africa, Research development grants for Y-rated Researchers Grant no. 93360 awarded to MM. The funding body had no role in the study design, collection, analysis, and interpretation of data or in writing the manuscript.

\section{Availability of data}

The data that support the findings of this study are available on request from the corresponding author [CK]. The data are not publicly available due to them containing information that could compromise research participant privacy/consent.

\section{Authors' contributions}

The work presented in the article was carried out in collaboration between all authors. $\mathrm{CK}, \mathrm{PVH}, \mathrm{EH}, \mathrm{ME}, \mathrm{MU}$ and $\mathrm{MM}$ made substantial contributions to the conception or design of the work. BG was responsible for exome sequencing data analysis. CK, BG, MU, MM and ME made significant contributions to the interpretation of data. CK and BG performed all laboratory experiments. CK, BG, MU and MM drafted the manuscript. All authors critically revised the final manuscript. All authors read and approved the final manuscript. All authors agreed to be accountable for all aspects of the work in ensuring that questions related to the accuracy or integrity of any part of the work are appropriately investigated and resolved.

\section{Competing interests}

The authors declare that they have no competing interests.

\section{Consent for publication}

Consent was obtained from the parents for publication and to include photographs in this article.

\section{Ethics approval and consent to participate}

The study was approved by the Health Research Ethics Committee of Stellenbosch University (study no. N13/05/075). Parents gave informed consent to participate in the study, which included the genetic evaluation of their children. The study adhered to the ethical guidelines as set out in the "Declaration of Helsinki, 2013" [20].

\section{Publisher's Note}

Springer Nature remains neutral with regard to jurisdictional claims in published maps and institutional affiliations.

\section{Author details}

${ }^{1}$ SA MRC Centre for Tuberculosis Research, DST/NRF Centre of Excellence for Biomedical Tuberculosis Research, Division of Molecular Biology and Human Genetics, Faculty of Medicine and Health Sciences, Stellenbosch University, P.O. Box 241, Cape Town 8000, South Africa. ${ }^{2}$ National Health Laboratory Service, Immunology Unit, Division of Medical Microbiology, Department of Pathology, Tygerberg Hospital, Stellenbosch University, Cape Town, South
Africa. ${ }^{3}$ Department of Paediatrics and Child Health, Faculty of Medicine and Health Sciences, Stellenbosch University, Cape Town, South Africa.

Received: 18 July 2016 Accepted: 2 March 2017

Published online: 14 March 2017

\section{References}

1. Stankler L, Lloyd D, Pollitt RJ, Gray ES, Thom H, Russell G. Unexplained diarrhoea and failure to thrive in 2 siblings with unusual facies and abnormal scalp hair shafts: a new syndrome. Arch Dis Child. 1982;57:212-6.

2. Fabre A, André N, Breton A, Broué P, Badens C, Roquelaure B. Intractable diarrhea with "phenotypic anomalies" and tricho-hepato-enteric syndrome: two names for the same disorder. Am J Med Genet A. 2007:143A:584-8.

3. Girault D, Goulet O, Le Deist F, Brousse N, Colomb V, Césarini JP, et al. Intractable infant diarrhea associated with phenotypic abnormalities and immunodeficiency. J Pediatr. 1994;125:36-42.

4. Verloes A, Lombet J, Lambert Y, Hubert AF, Deprez M, Fridman V, et al. Tricho-hepato-enteric syndrome: further delineation of a distinct syndrome with neonatal hemochromatosis phenotype, intractable diarrhea, and hair anomalies. Am J Med Genet. 1997:68:391-5.

5. Kotecha UH, Movva S, Puri RD, Verma IC. Trichohepatoenteric syndrome: founder mutation in asian indians. Mol Syndromol. 2012;3:89-93.

6. Hartley JL, Zachos NC, Dawood B, Donowitz M, Forman J, Pollitt RJ, et al. Mutations in TTC37 cause trichohepatoenteric syndrome (phenotypic diarrhea of infancy). Gastroenterology. 2010;138:2388-98. 2398-2.

7. Goulet O, Vinson C, Roquelaure B, Brousse N, Bodemer C, Cézard J-P. Syndromic (phenotypic) diarrhea in early infancy. Orphanet J Rare Dis. 2008;3:6.

8. Costa-Carvalho BT, Grumach AS, Franco JL, Espinosa-Rosales FJ, Leiva LE, King $A$, et al. Attending to warning signs of primary immunodeficiency diseases across the range of clinical practice. J Clin Immunol. 2014;34:10-22.

9. Subbarayan A, Colarusso G, Hughes SM, Gennery AR, Slatter M, Cant AJ, et al. Clinical features that identify children with primary immunodeficiency diseases. Pediatrics. 2011;127:810-6.

10. Lee W-I, Huang J-L, Chen C-C, Lin J-L, Wu R-C, Jaing T-H, et al. Identifying Mutations of the Tetratricopeptide Repeat Domain 37 (TTC37) Gene in Infants With Intractable Diarrhea and a Comparison of Asian and Non-Asian Phenotype and Genotype: A Global Case-report Study of a Well-Defined Syndrome With Immunodeficiency. Medicine (Baltimore). 2016;95:e2918.

11. Carneiro-Sampaio M, Jacob CMA, Leone CR. A proposal of warning signs for primary immunodeficiencies in the first year of life. Pediatr Allergy Immunol. 2011;22:345-6.

12. Fabre A, Martinez-Vinson C, Goulet O, Badens C. Syndromic diarrhea/Trichohepato-enteric syndrome. Orphanet J Rare Dis. 2013;8:5.

13. de Vries E, Visser DM, van Dongen JJ, Jacobs CJ, Hoekstra JH, van Tol MJ. Oligoclonal gammopathy in phenotypic diarrhea. J Pediatr Gastroenterol Nutr. 2000;30:349-50.

14. Fabre A, Charroux B, Martinez-Vinson C, Roquelaure B, Odul E, Sayar E, et al. SKIV2L mutations cause syndromic diarrhea, or trichohepatoenteric syndrome. Am J Hum Genet. 2012;90:689-92.

15. Rider NL, Boisson B, Jyonouchi S, Hanson EP, Rosenzweig SD, Cassanova J-L, et al. Novel TTC37 mutations in a patient with immunodeficiency without diarrhea: extending the phenotype of Trichohepatoenteric syndrome. Front Pediatr. 2015;3:2

16. Zheng B, Pan J, Jin Y, Wang C, Liu Z. Targeted next-generation sequencing identification of a novel missense mutation of the SKIV2L gene in a patient with trichohepatoenteric syndrome. Mol Med Rep. 2016;14:2107-10.

17. Anderson JS, Parker RP. The $3^{\prime}$ to $5^{\prime}$ degradation of yeast mRNAs is a general mechanism for mRNA turnover that requires the SKI2 DEVH box protein and $3^{\prime}$ to $5^{\prime}$ exonucleases of the exosome complex. EMBO J. 1998; 17:1497-506.

18. van Hoof A, Frischmeyer PA, Dietz HC, Parker R. Exosome-mediated recognition and degradation of mRNAs lacking a termination codon. Science. 2002;295:2262-4.

19. Orban TI, Izaurralde E. Decay of mRNAs targeted by RISC requires XRN1, the Ski complex, and the exosome. RNA. 2005;11:459-69.

20. World Medical Association. World Medical Association Declaration of Helsinki: ethical principles for medical research involving human subjects. JAMA. 2013;310:2191-4.

21. Glanzmann B, Herbst $\mathrm{H}$, Kinnear CJ, Möller M, Gamieldien J Bardien S. A new tool for prioritization of sequence variants from whole exome 
sequencing data. Source Code Biol. Med. [Internet]. 2016 [cited 4 Jul 2016]; 11. Available from: http://www.ncbi.nlm.nih.gov/pmc/articles/PMC4929716/.

22. Purcell S, Neale B, Todd-Brown K, Thomas L, Ferreira MAR, Bender D, et al. PLINK: a tool set for whole-genome association and population-based linkage analyses. Am J Hum Genet. 2007;81:559-75.

23. Richards S, Aziz N, Bale S, Bick D, Das S, Gastier-Foster J, et al. Standards and guidelines for the interpretation of sequence variants: a joint consensus recommendation of the American College of Medical Genetics and Genomics and the Association for Molecular Pathology. Genet Med. 2015; 17:405-24.

24. Venselaar $H$, Te Beek TAH, Kuipers RKP, Hekkelman ML, Vriend G. Protein structure analysis of mutations causing inheritable diseases. An e-Science approach with life scientist friendly interfaces. BMC Bioinformatics. 2010;11:548.

25. Orange JS. Natural killer cell deficiency. J Allergy Clin Immunol. 2013; 132:515-26.

26. Oz-Levi D, Weiss B, Lahad A, Greenberger S, Pode-Shakked B, Somech R, et al. Exome sequencing as a differential diagnosis tool: resolving mild trichohepatoenteric syndrome. Clin Genet. 2015;87:602-3.

27. Stark Z, Tan TY, Chong B, Brett GR, Yap P, Walsh M, et al. A prospective evaluation of whole-exome sequencing as a first-tier molecular test in infants with suspected monogenic disorders. Genet Med. 2016;18:1090-6.

28. Tarailo-Graovac M, Shyr C, Ross CJ, Horvath GA, Salvarinova R, Ye XC, et al. Exome sequencing and the management of neurometabolic disorders. $\mathrm{N}$ Engl J Med. 2016:374:2246-55.

29. Need AC, Shashi V, Hitomi Y, Schoch K, Shianna KV, McDonald MT, et al. Clinical application of exome sequencing in undiagnosed genetic conditions. J Med Genet. 2012;49:353-61.

\section{Submit your next manuscript to BioMed Central and we will help you at every step:}

- We accept pre-submission inquiries

- Our selector tool helps you to find the most relevant journal

- We provide round the clock customer support

- Convenient online submission

- Thorough peer review

- Inclusion in PubMed and all major indexing services

- Maximum visibility for your research

Submit your manuscript at www.biomedcentral.com/submit 